\title{
Preferential Compatibility in Streptomyces violaceoruber
}

\author{
By S. G. BRADLEY AND D. L. ANDERSON \\ Department of Bacteriology, University of Minnesota, Minneapolis 14, \\ Minnesota, U.S.A.
}

(Received 15 March 1960)

\begin{abstract}
SUMMARY
A compatibility system, or system of relative mating potency, has been found among mutants of Streptomyces violaceoruber ( $S$. coelicolor). Certain combinations of growth factor-dependent mutants failed to form nutritionally independent (prototrophic) recombinants. Incompatibility was not the result of inability of identical mutant genes to interact, failure of hyphae to fuse, or inhibition of prototroph development by incompatible mixtures. No infective or diffusible factors were found which induced or suppressed fertility. The yield of recombinants was affected by the relative growth rates of the two parental types, the time elapsed between the planting of each parent and the relative and absolute numbers of the parents. Sporogenesis was not a prerequisite for recombination.
\end{abstract}

\section{INTRODUCTION}

Gene recombination in Streptomyces violaceoruber (S. coelicolor) was reported by Sermonti \& Spada-Sermonti (1955, 1956). They isolated nonparental types from mixed growth of complementary multiple auxotrophic mutants of $S$. violaceoruber. Similar systems were reported in $S$. griseus (Bradley \& Lederberg, 1956), in $S$. violaceoruber (Bradley, 1957; Hopwood, 1959), in $S$. violaceoruber and $S$. fradiae (Szybalski \& Braendle, 1956), in S. rimosus (Alikhanian \& Mindlin, 1957), and in $S$. griseoflavus (Saito, 1958). Although the recombinational mechanism has been studied extensively, little consideration has been given to preferential mating. The occurrence of incompatibility in Streptomyces was expected inasmuch as incompatibility reactions have been established in several species of fungi and bacteria. For example, a non-filterable contagious factor that could be transmitted to other cells by direct contact was found to be responsible for fertility in Escherichia coli strain K-12 (Lederberg, Cavalli \& Lederberg, 1952). In Neurospora crassa heterokaryotic incompatibility was manifested primarily in the vegetative hyphae (Garnjobst \& Wilson, 1956). Hyphal fusion in non-fertile combinations of $N$. crassa was followed by a series of visible changes involving irreversible destruction of fusing cells. At first, all strains of $S$. violaceoruber tested interacted to form prototrophic progeny. Subsequently, growth factor-dependent mutants unable to interact with other variants were found and evidence for the existence of a compatibility system controlling recombination in $S$. violaceoruber was presented (Bradley \& Anderson, 1958). The purpose of this study was to determine and analyse the genetic and environmental factors controlling recombination in $S$. violaceoruber. 


\section{Glossary}

Prototroph. An organism which grew on minimal medium composed only of glucose, inorganic salts and water.

Auxotroph. An organism which did not grow on minimal medium unless supplemented with one or more specific growth-factors.

Recombination. The process by which two strains interacted to produce offspring phenotypically unlike the parents. A recombinant might be either prototrophic or auxotrophic.

Compatibility or fertility. The experimentally observed production of recombinants.

Heterokaryon. An organism having genetically different nuclei within a common cytoplasm.

Sterility. Inability to detect production of recombinants.

Streptomyces violaceoruber. This is a species which has often been erroneously designated as Streptomyces coelicolor. S. violaceoruber forms spirals, utilizes L-rhamnose and raffinose, and produces blue pigment on many media. $S$. coelicolor forms straight spore chains, does not utilize L-rhamnose and raffinose, and does not produce blue pigment on most media.

\section{METHODS}

The strains used in this study were auxotrophic (growth factor-dependent) mutants derived from a single wild-type strain of Streptomyces vinaceus and three wild-type strains of $S$. violaceoruber (Table 1). These latter organisms were previously designated $S$. coelicolor; Kutzner \& Waksman (1959), however, pointed out recently that the correct epithet is $S$. violaceoruber.

Stock cultures were maintained on complete medium agar of the following composition. Solution I: glucose, 20 g.; Difco yeast extract (Difco Laboratories, Inc., Detroit, Michigan, U.S.A.) 1 g.; Difco Casamino acids, 1 g.; Difco agar, 15 g.; $\mathrm{MgSO}_{4} .7 \mathrm{H}_{2} \mathrm{O}, 0.5 \mathrm{~g}$; deionized water, $500 \mathrm{ml}$. Solution II: $\mathrm{KNO}_{3}, 2 \mathrm{~g}$.; $\mathrm{Na}_{2} \mathrm{HPO}_{4}$,


with that of complete medium except the former lacked yeast extract and Casamino acids. In the preparation of both complete medium and minimal medium, the two solutions were autoclaved separately and mixed before the media were dispensed. The complex medium was composed of: Difco peptone, 5 g.; Difco yeast extract, 3 g.; glucose, 1 g.; Difco Casitone, 1 g.; Armour beef extract, 2 g.; Difco agar, 15 g.; deionized water, 11 . Comparable liquid media were made by omitting the agar. All media were autoclaved at $121^{\circ}$ for $20 \mathrm{~min}$.

Auxotrophic mutants were detected by the following method. Spores which survived ultraviolet irradiation (99\% kill) were cultured on complete medium; the resulting growth was transferred to minimal and complete media by replica plating (Lederberg \& Lederberg, 1952); colonies which yielded growth on complete medium but not on minimal medium were tentatively designated as growth factor-dependent variants. The auxanographic technique was employed in characterizing all mutants. Susceptibility of natural variants or selected variants to actinophage $P 7$ was also used as a genetic marker; phage-resistant strains were selected by planting $10^{6}-10^{8}$ 
plating units of the phage-sensitive organism on complex medium seeded with $10^{7}-10^{9}$ actinophage particles. Colonies arising on these plates were picked and streaked on complex medium containing an excess of actinophage to verify resistance. Susceptibility to actinophage was determined by the cross-streak technique: a single streak $8 \mathrm{~cm}$. long, of phage suspension $\left(0.01 \mathrm{ml}\right.$. of a $10^{8}$ phage particles/ ml. suspension) was made on complex medium agar and allowed to dry; streptomycetes to be tested were cross-streaked against this single phage streak. Failure of the test organisms to grow at the intersections indicated susceptibility to the phage. This method permitted rapid classification of a large number of progeny. Morphological differences inherent in the strains, such as colonial texture and pigmentation, were noted but not considered reliable genetic markers.

\section{Table 1. Principal stocks}

\begin{tabular}{|c|c|c|}
\hline Strain & Origin & Phenotype* \\
\hline S 199 & $\begin{array}{l}\text { S. violaceoruber } \\
\text { Nature, Wisc. Soil } \\
\text { Sample }\end{array}$ & Prototrophic Prg \\
\hline S202 & $\mathbf{S} 199$ & 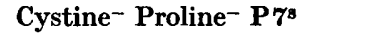 \\
\hline S203 & S 199 & Uracil- $^{-}$arginine- $\mathbf{P}^{\mathrm{s}}$ \\
\hline $\mathbf{S 2 0 4}$ & S 199 & Methionine- P $7^{\mathrm{s}}$ \\
\hline Sermonti 1 & $\begin{array}{l}\text { S. violaceoruber } \\
\text { G. Sermonti, Rome, } \\
\text { Italy }\end{array}$ & Prototrophic Prs \\
\hline S205 & Sermonti 1 & Histidine $^{-}$P $\boldsymbol{7}^{\mathrm{s}}$ \\
\hline Sermonti 23 & Sermonti 1 & Methionine ${ }^{-}$histidine ${ }^{-} \mathbf{P} 7^{8}$ \\
\hline Sermonti 30 & Sermonti 1 & Nicotinic acid- \\
\hline S16 & $\begin{array}{l}\text { S. violaceoruber } \\
\text { NRRL-B-1257 U.S. } \\
\text { Dept. Agric. Peoria, } \\
\text { Ill. }\end{array}$ & Prototrophic P7r \\
\hline S206 & S16 & Arginine $^{-} \mathbf{P} \boldsymbol{\gamma}^{\mathbf{r}}$ \\
\hline $\mathrm{S} 207$ & S 16 & Hydroxy-proline- P Pr \\
\hline S125 & S 16 & Methionine $-\mathbf{P} \mathbf{7}^{\mathrm{r}}$ \\
\hline S208 & $\begin{array}{l}\text { S. vinaceus } \\
\text { Nature, Minn. Soil } \\
\text { Sample }\end{array}$ & Prototrophic P $\boldsymbol{\gamma}^{\mathrm{r}}$ \\
\hline S216 & S208 & Aspartic acid- \\
\hline S217 & $\mathrm{S} 208$ & Glutamic acid- \\
\hline S229 & S207 & Arginine $^{-}$alanine $^{-} \mathbf{P} \mathbf{7}^{\mathrm{r}}$ \\
\hline S230 & S207 & Arginine $^{-}$glutamic acid $-\mathbf{P} \gamma^{x}$ \\
\hline
\end{tabular}

* Abbreviations: $\mathbf{P} \mathbf{\gamma}^{\mathrm{s}}$ denotes susceptibility to actinophage $\mathbf{P r} ; \mathbf{P} \mathbf{\gamma}^{\mathrm{r}}$ denotes resistance to actinophage P7; prototrophic denotes growth-factor independence; cystine ${ }^{-}$proline $^{-}$, etc., denote requirements for cystine and proline, etc., for growth.

Inocula for crossing experiments were prepared by harvesting aerial hyphae from stock plate cultures and suspending these hyphae in sterile deionized water. Preparation of inocula of mutants producing little or no aerial hyphae was more difficult because it was necessary to use vegetative mycelia. Potter-Elvehjem tissue homogenizers were used to reduce coarse suspensions of vegetative mycelia to homogeneous suspensions.

Recombinants were obtained by seeding approximately $10^{5}$ plating units of each parent together on complex medium, incubating these mixed cultures at $30^{\circ}$ for 6-14 days, and transferring samples to minimal and complete media by replica 
plating. Mixed growth was transferred to complete medium in order to verify the viability of both parental strains in the mixture and to provide an estimate of the relative growth of the parents on the crossing medium. Replica plates were scored for prototrophs after incubation at $30^{\circ}$ for 3-5 days. In addition to their ability to grow on minimal medium, prototrophic colonies grew faster and produced greater quantities of aerial hyphae on all media than did the parental strains.

\section{RESULTS}

\section{Compatibility system}

Eleven auxotrophic mutants of Streptomyces violaceoruber were grown in most of the possible pairwise combinations on complex medium agar. Strain S207 (a derivative of wild-type strain S16) was not fertile with strains S202, S 203 and S 204 (descendants of wild-type strain S199) or with strains Sermonti 23 and Sermonti 30, which were derived from wild-type strain Sermonti 1 (Table 2). Strain S 207 was

\section{Table 2. The compatibility system}



Two complementary auxotrophic strains were grown together on complex medium at $30^{\circ}$. After 5-14 days, a sample of mixed growth was transferred to minimal medium by replica plating. Prototroph production was rated as:

$$
\begin{aligned}
+ & =5-25 \text { prototrophs/plate. } \\
++ & =10-50 \text { prototrophs/plate. } \\
+++ & =30-200 \text { prototrophs/plate. } \\
++++ & =\text { over } 200 \text { prototrophs/plate. } \\
* & =\text { not tested. } \\
+ & =\text { not testable because these strains have a common metabolic defect. } \\
- & =\text { no prototrophs produced. }
\end{aligned}
$$

fertile, however, with strains S125 and S206, which were derived from wild type strain S16. These results, and results of other crosses, supported the hypothesis that the closer the relationship between two organisms the higher was the fertility. This was not always true, however, for strain $\mathrm{S} 125$ produced more recombinants with strains S202 and S203 than with strains S206 or S207. Derivatives of S. vinaceus strain S 208 were sterile in combination with all $S$. violaceoruber strains so far as they were tested, and therefore provided no information about this incompatibility system. 


\section{Mechanism of incompatibility}

It was conceivable that mixed growth of incompatible strains inhibited growth of prototrophs. To test this possibility, fragmented aerial hyphae from prototrophic wild-type strain S199 were added to mixed inocula of strains S 205 and S207 (an incompatible combination) and the mixture was plated on complex medium. After incubation at $30^{\circ}$ for 8 days, a sample of mixed growth was transferred to minimal medium by replica plating in an attempt to recover the $\mathbf{S 1 9 9}$ prototrophs added originally. Prototrophic colonies of strain S199 were clearly visible 5 days after incubation of the replica plates at $30^{\circ}$ (Table 3). It was concluded that mixed growth of strains S207 and S205 did not inhibit the development of S199 prototrophs; therefore, it was unlikely that suppression of prototroph development by non-fertile combinations was responsible for incompatibility.

Table 3. Recovery of $S 199$ prototrophs from mixed growth of $S 207+S 205$

\begin{tabular}{|c|c|c|c|}
\hline \multicolumn{3}{|c|}{ Inoculum size in plating units } & \multirow{2}{*}{$\begin{array}{c}\text { Average } \\
\text { number of } \\
\text { recovered } \\
\text { prototrophs } \\
\text { (S 199) per } \\
\text { plate }\end{array}$} \\
\hline S207 & S205 & S 199 & \\
\hline $5 \times 10^{6}$ & $5 \times 10^{6}$ & $10^{3}$ & 230 \\
\hline $5 \times 10^{8}$ & $5 \times 10^{6}$ & $10^{2}$ & 22 \\
\hline $5 \times 10^{8}$ & $5 \times 10^{8}$ & 10 & $\mathbf{2}$ \\
\hline $5 \times 10^{6}$ & $5 \times 10^{6}$ & $\mathbf{0}$ & O \\
\hline $5 \times 10^{6}$ & 0 & $10^{2}$ & $\mathbf{2 0}$ \\
\hline $\mathbf{0}$ & $5 \times 10^{6}$ & $10^{2}$ & 18 \\
\hline 0 & $5 \times 10^{6}$ & 0 & $\mathbf{0}$ \\
\hline $5 \times 10^{6}$ & $\mathbf{0}$ & $\mathbf{0}$ & $\mathbf{0}$ \\
\hline
\end{tabular}

Aerial hyphae from prototrophic wild-type strain S199 were added to mixed inocula of S205 and $\mathrm{S} 207$ (an incompatible combination) and the mixture was plated to complex medium. After incubation at $30^{\circ}$ for 8 days, a sample of mixed growth was transferred to minimal medium by replica plating in an attempt to recover the $\mathbf{S} 199$ prototrophs added originally. Replica plates were scored for the presence of $\mathrm{S} 199$ colonies after incubation for 5 days at $30^{\circ}$.

A microscopic search for hyphal fusion in crosses between mutants involved in the compatibility system was made, using a maximum total magnification of $\times \mathbf{2 0 0 0}$. To detect hyphal fusion, two mutants were grown in close proximity on complete medium. When hyphal tips of the two strains were close enough for conjugation, a small block of agar including parallel streaks of the organisms was cut from the plate and carefully melted beneath a coverslip on a glass microscope slide. Search for hyphal fusion was conducted only in areas where the positions of both mutants could be definitely determined. Hyphal fusion occurred as well in non-fertile combinations as in fertile combinations. Fusion of hyphae was observed in the fertile combinations S206+S207, S 206+S 204, S206+S125 and S203+ Sermonti 23 , and also in the non-fertile combinations S207+S205, S207+S204, and S207+ S203. Incompatibility, therefore, did not result from inability of hyphae of incompatible pairs to fuse, since hyphal fusion occurred both in compatible and incompatible combinations.

To test the possibility that fertile combinations might produce a filtrable fertility factor which would induce compatibility in sterile combinations, the fertile combina- 
tion S206+S125 and the incompatible combination S205+S207 were grown in complex medium broth on opposite sides of a fritted glass filter. The partitioned cultures were incubated at $30^{\circ}$ for 9-16 days and the liquid medium was exchanged across the filter daily by using suction from a water aspirator. Following incubation, the growth mixtures were removed from both sides of the filter, harvested by centrifugation, and washed twice with sterile deionized water. The mixtures were then suspended in sterile deionized water and transferred aseptically to tissue homogenizers for dispersion to provide homogeneous inoculative suspensions. To determine whether prototrophs were formed, samples of each of the mixed growth suspensions were plated to minimal and complete media. The compatible $\mathrm{S} 206+\mathrm{S} 125$ combination was fertile under these conditions, but the incompatible S $207+$ S 205 combination was sterile (Table 4).

Table 4. Tests for a filtrable fertility factor

\begin{tabular}{|c|c|c|c|c|}
\hline $\begin{array}{l}\text { Pair } 1 \text { and pair } 2 \\
\text { separated by }\end{array}$ & Pair 1 & $\begin{array}{c}\text { Average } \\
\text { number of } \\
\text { prototrophs/ } \\
\text { plate }\end{array}$ & Pair 2 & $\begin{array}{c}\text { Average } \\
\text { number of } \\
\text { prototrophs/ } \\
\text { plate }\end{array}$ \\
\hline Fritted glass & $\mathrm{S} 206+\mathrm{S} 125$ & 250 & $\mathrm{~S} 207+\mathrm{S} 205$ & 0 \\
\hline Cellophan & $\begin{array}{c}\text { S 206 + S 125 } \\
\text { (bottom) } \\
\text { S 206 + S } 125 \\
\text { (top) }\end{array}$ & $\begin{array}{r}13 \\
5\end{array}$ & $\begin{array}{c}\mathrm{S} 207+\mathrm{S} 205 \\
\text { (top) } \\
\mathrm{S} 207+\mathrm{S} 205 \\
\text { (bottom) }\end{array}$ & $\begin{array}{l}0 \\
0\end{array}$ \\
\hline
\end{tabular}

The fertile combination S206+S125 and the incompatible combination S207+S205 were grown on opposite sides of a fritted glass filter or a cellophan membrane. Following incubation at $30^{\circ}$, samples of mixed growth from both sides of the fritted filter (or cellophan membrane) were transferred to minimal medium. Minimal medium plates were scored for prototroph production after incubation at $30^{\circ}$ for 3-5 days.

Additionally, the fertile combination S206 +S125 and the incompatible combination $\mathrm{S207}+\mathrm{S} 205$ were grown on opposite sides of a cellophan membrane, with inocula of approximately $5 \times 10^{6}$ plating units of each parental strain. After incubation at $30^{\circ}$ for 8 days, samples of mixed growth from each side of the membrane were suspended in deionized water, dispersed in a tissue homogenizer, and plated on minimal and complete media to determine prototroph production. All compatible pairs yielded prototrophs but incompatible combinations remained sterile. The results of this experiment further confirmed the hypothesis that fertile combinations did not produce filtrable factors capable of inducing fertility in incompatible combinations.

An infective factor, similar to the contagious $\mathbf{F}+$ factor in Escherichia coli might control fertility in the compatibility system. To test this possibility, two complementary auxotrophic strains were grown in mixed culture on complex medium for 8 days at $30^{\circ}$. Then one or both of the parents were recovered from the mixed growth by transferring (replica plating) the mixed growth to minimal media supplemented with the specific nutritional requirements of the organisms being recovered. The parents, re-isolated after growth in a fertile combination, were then crossed to other strains to determine whether factors were acquired which modified ability to be successfully crossed or induced fertility in incompatible pairs. Non-fertile combinations, however, remained sterile even though one or both parents 
had been previously grown in fertile combinations (Table 5). The results, therefore, indicated that no contagious fertility factor was responsible for compatibility in Streptomyces violaceoruber and that prior association with a compatible partner did not increase the recombinational frequency in subsequent crosses, Conversely, Braendle, Gardiner \& Szybalski (1959), found that complementary 'secondary parental isolates' yielded more heterokaryons/plate than 'primary parental isolates'.

Table 5. Tests for a contagious fertility factor

\begin{tabular}{|c|c|}
\hline Combination & $\begin{array}{c}\text { Average number } \\
\text { of prototrophs/ } \\
\text { plate }\end{array}$ \\
\hline S207+S206 (S205) & 23 \\
\hline $\mathrm{S} 207+\mathrm{S} 205(\mathrm{~S} 206)$ & $\mathbf{0}$ \\
\hline S205+S206 (S207) & 26 \\
\hline $\mathrm{S} 205+\mathrm{S} 207(\mathrm{~S} 206)$ & $\mathbf{0}$ \\
\hline S205+S207 (S125) & 0 \\
\hline $\mathrm{S} 205(\mathrm{~S} 206)+\mathrm{S} 207(\mathrm{~S} 125)$ & $\mathbf{0}$ \\
\hline
\end{tabular}

Symbolism: S206 (S205) denotes that S206 had been grown in combination with S 205 and then re-isolated.

Two complementary auxotrophic strains were grown together on complex medium at $30^{\circ}$. After 8 days, one or both of the parents were recovered from the mixed growth by replica plating the mixed growth to minimal media supplemented with the specific nutritional requirements of the organisms being recovered. Recovered strains were crossed to other strains as desired by growing the two organisms in mixed culture on complex medium at $30^{\circ}$. After 8 days, samples of mixed growth were transferred to minimal medium by replica plating to determine prototroph production.

Table 6. Effect of age of the mixed culture on prototroph production

\begin{tabular}{ccc}
$\begin{array}{c}\text { Days of } \\
\text { incubation }\end{array}$ & \multicolumn{2}{c}{$\begin{array}{c}\text { Average number of } \\
\text { prototrophs/plate }\end{array}$} \\
& S206+S125 & $\begin{array}{c}\text { S203+Ser- } \\
\text { monti 23 }\end{array}$ \\
2 & 0 & 0 \\
3 & 0 & 0 \\
4 & 20 & 93 \\
5 & 19 & 106 \\
6 & 27 & 103 \\
7 & 31 & 131 \\
8 & 30 & 125 \\
9 & 33 & 142 \\
10 & 28 & 136 \\
& 40 & 129
\end{tabular}

Pairwise mixtures of complementary auxotrophic strains were grown on complex medium at $30^{\circ}$. After $24 \mathrm{hr}$. and daily thereafter for 10 days, duplicate samples of mixed growth were transferred to minimal medium by replica plating. Replica plates were scored for prototroph production after 3-5 days incubation at $30^{\circ}$.

The fertile combinations S206+S125 and S203+Sermonti 23 were grown on complex medium agar to determine the minimum time interval between mixing of the mutant strains and detection of prototrophic progeny. Also, it was desirable to know whether the total number of prototrophs produced from a fertile combination increased after the time of first detection. Twenty-four hours after mixing the mutants, and daily thereafter for 10 days, duplicate samples of mixed growth 
were transferred to minimal and complete media by replica plating to determine prototroph production. Prototrophs were not detected until the mixed cultures had been incubated for 3 days; from the third day of incubation to the tenth day of incubation, the quantity of prototrophs produced remained constant or increased slightly (Table 6).

\section{Effect of inoculum size on prototroph production}

Prototroph production was also affected by the inoculum size of each of the auxotrophic mutants involved in a cross and by the relative growth rates of the auxotrophic parents. In the Sermonti $23+\mathrm{S} 203$ combination, only c. 2 prototrophs/ plate were produced when the inoculum of the former strain (approximately $5 \times 10^{6}$ plating units) exceeded greatly the inoculum of the latter strain (approximately $5 \times 10^{2}$ plating units). No prototrophs were produced when the inoculum of strain S 203 greatly exceeded the inoculum of strain Sermonti 23. An average of 10 prototrophs/plate were formed when the inocula of both parents were approximately $5 \times 10^{2}$ plating units, and an average of 23 prototrophs/plate were produced when the inocula of both parents were approximately $5 \times 10^{6}$ plating units.

Decrease in prototroph production caused by a difference in the growth rates of the auxotrophic parents could be countered in most cases by allowing the slow growing parent to grow for 1-2 days before addition of the fast growing parent. When the growth rates of both parents were about equal, addition of one of the parents 1-3 days after the other parent did not appreciably affect prototroph production. When addition of the inoculum of the second parent was delayed longer than 3 days, no prototrophs were formed. These results indicated either that established growth no longer had the ability to form prototrophs or that an antibiotic was produced which killed the delayed inoculum. The latter alternative was disproved by demonstrating that both parental types could be recovered.

\section{Yield of recombinants from aerial hyphae and vegetative hyphae}

It was of interest to ascertain whether prototrophic recombinants could be recovered from both aerial and vegetative hyphae and to determine which of these sources produced the greatest quantity of prototrophs. As a representative fertile combination, strains S203 and Sermonti 23 were grown in mixed culture on complex medium at $30^{\circ}$ for 10 days. Suspensions of aerial and vegetative hyphae were then prepared from the mixed growth and samples of these suspensions were plated on minimal and complete media. Relatively homogeneous suspensions for inoculations were prepared by thoroughly grinding the coarse hyphal suspensions in sterile Potter-Elvehjem tissue homogenizers. The number of plating units $/ \mathrm{ml}$. of the aerial and vegetative inoculum suspensions were calculated from counts obtained with a Petroff-Hauser bacteria counter. Plates were scored for prototroph formation after incubation at $30^{\circ}$ for 4 days. An average of $2.5 \times 10^{-5}$ prototrophs/plating unit of mixed growth were obtained from the aerial hyphae of the Sermonti $23+\mathrm{S} 203$ combination and an average of $5.6 \times 10^{-5}$ prototrophs/plating unit were produced from the vegetative hyphae. The results of these experiments showed that in the compatible combination S203 + Sermonti 23, approximately twice as many prototrophs were produced in the vegetative hyphae as in the aerial hyphae, per plating unit of mixed growth. 


\section{Phage susceptibility tests on prototrophic recombinants}

Prototrophic progeny from several combinations in the compatibility system were tested for susceptibility to actinophage P7. Phage susceptibility of 106 prototrophic colonies from crosses between strains S 206 and S 205, and of 115 prototrophic colonies from crosses between strains S206 and S 204, was determined using the cross-streak technique. In both crosses, susceptibility to actinophage $\mathbf{P} 7$ could segregate since strain S206 was resistant to phage $P 7$ and strains S205 and S204 were sensitive. All prototrophs tested from the S 205 ( $\mathrm{P} 7$ sensitive $) \times \mathrm{S} 206$ (P7 resistant) cross and the S204 (P7 sensitive) $\times \mathrm{S} 206$ ( $\mathrm{P} 7$ resistant) cross were found to be sensitive. These results suggested that the recombinants were heterokaryons, heterozygous diploids, or haploid gene recombinants in which actinophage susceptibility was linked closely to a selected character.

\section{DISCUSSION}

Certain nutritionally dependent mutants of Streptomyces violaceoruber in pairwise mixtures failed to form growth factor-independent prototrophic colonies (Bradley \& Anderson, 1958). Failure of these combinations to produce prototrophs was not the result of inability of identical mutant genes determining nutritional requirements to interact, because diverse markers were used. Moreover, two strains, each unable to interact with a third strain, were compatible with each other, a situation which would have been impossible if the genes preventing heterokaryon formation were identical. Therefore a specific compatibility system exists which controls recombination. The possibility that prototroph development was inhibited in an incompatible combination was ruled out by the successful recovery of added strain S199 prototrophs from the incompatible S207+S 205 mixture, following incubation. Hyphal fusion occurred in non-fertile as well as in fertile combinations, so inability of hyphae of incompatible pairs to fuse was not responsible for incompatibility. The barrier to compatibility might operate by preventing nuclear exchange or by preventing establishment of balanced nuclear ratios. The former hypothesis seems probable in view of the finding that fusion of hyphae in non-fertile combinations of Neurospora crassa is followed by destruction of fusing cells (Garnjobst \& Wilson, 1956). The difficulty of cytological studies following hyphal fusion in sterile combinations of $S$. violaceoruber prevents positive comparison, however, of the reaction occurring in $S$. violaceoruber with the irreversible reaction occurring in $N$. crassa. No filtrable or infective fertility factors were demonstrated in the compatibility system described here.

The strains of Streptomyces violaceoruber studied here can be divided into three groups (designated $\mathbf{A}, \mathbf{B}$, and $\mathrm{C}$ ) on the basis of their compatibility behaviour. Strains in group B produce prototrophs in combination with all other mutants of $S$. violaceoruber not having the same metabolic defect. Mutants belonging to group A are compatible with members of group B but not with members of group C. Groups A and B include mutant strains derived from wild type strain S 16. Group A is exemplified by strain S207 and group B by strain S125. Group C, which contains mutants derived from wild-type strains Sermonti 1 and S199, is typified by strain $S 203$. The general pattern of the compatibility system controlling recombination in $S$. violaceoruber may be summarized as follows: $\mathbf{A}+\mathbf{B}, \mathbf{B}+\mathbf{C}, \mathbf{A}+\mathbf{A}, \mathbf{B}+\mathbf{B}$, and 
$\mathrm{C}+\mathrm{C}$ are fertile; $\mathrm{A}+\mathrm{C}$ is not fertile. The data indicate that two or more factors may control heterokaryotic compatibility in $S$. violaceoruber The genotypes of $\mathrm{A}, \mathrm{B}$, and $\mathrm{C}$ can be symbolized as $a_{1} b_{1}, a_{1} b_{2}$, and $a_{2} b_{2}$, respectively. For mating to occur, the two strains involved must carry identical factors at one or both of the two loci. A fourth genotype $a_{2} b_{1}$, which has not yet been discovered, is predicted by this hypothesis. Accordingly, this strain should mate with $\mathbf{A}$ and $\mathbf{C}$ but not with $\mathbf{B}$.

The compatibility described in this study represents interaction between three wild type strains of Streptomyces violaceoruber of different origin. Hence recombination was demonstrated between the derivatives of strains of diverse origin as well as between derivatives of a single wild type strain. These results differ from the observations of Braendle \& Szybalski (1957) who reported that all crosses between mutants of diverse origin, whether or not the parental strains were classified in the same species, were non-fertile, i.e. no recombinants were formed. Crosses between mutant derivatives of $S$. vinaceus and mutants derived from each of the wild type strains of $S$. violaceoruber were unsuccessful, but a cross between two mutants derived from the same strain of $S$. vinaceus was fertile (Table 2 ).

All incompatible reactions in the compatibility system involve Streptomyces violaceoruber strain S207, a descendant of wild type strain S16. Strain S207 was not fertile with derivatives of wild-type strains $\mathrm{S} 199$ (isolated from Wisconsin soil sample) and Sermonti 1 (provided by G. Sermonti). Strain S 207 was fertile, however, with other derivatives of strain S16, from which strain S207 itself was derived. These and other results supported the hypothesis that the greater the similarity between two organisms, the higher the fertility. Exceptions to this hypothesis, however, are exemplified by the high fertility of crosses between strain $\mathrm{S} 125$ (a derivative of S16) and mutants derived from wild-type strains S199 and Sermonti 1.

Fertile combinations of auxotrophic mutants of Streptomyces violaceoruber must be grown on complex medium for a period of at least 3 days before prototrophs can be detected. It is not known whether this lag represents a delay in hyphal fusion or a delay following hyphal fusion. From the third day of incubation to the tenth day of incubation the quantity of prototrophs produced remained fairly constant. Maximum prototroph production was reached on about the fifth day. Both aerial hyphae and vegetative hyphae yielded prototrophic recombinants. The vegetative hyphae produced approximately twice as many prototrophs as the aerial hyphae per plating unit of mixed growth. This established that sporogenesis was not a prerequisite for recombination.

This investigation was supported by a research grant E-1601 (C-2) from the National Institute of Allergy and Infectious Diseases, United States Public Health Service. Part of the work reported here is taken from a thesis submitted by D.L.A. as partial requirement for the Master of Science degree.

\section{REFERENCES}

Alikhanian, S. I. \& Mindlin, S. Z. (1957). Recombinations in Streptomyces rimosus. Nature, Lond. 180, 1208.

Bradley, S. G. (1957). Heterokaryosis in Streptomyces coelicolor. J. Bact. 73, 581.

Bradley, S. G. \& Anderson, D. L. (1958). Compatibility system controlling heterokaryon formation in Streptomyces coelicolor. Proc. Soc. exp. Biol., N.Y. 99, 476. 
Bradley, S. G. \& Lederberg, J. (1956). Heterokaryosis in Streptomyces. J. Bact. 72, 219.

Braende, D. H. \& SzYBalski, W. (1957). Genetic interaction among streptomyces: heterokaryosis and synkaryosis. Proc. nat. Acad. Sci., Wash. 43, 947.

Braendle, D. H., Gardiner, B. \& Szybalski, W. (1959). Heterokaryotic compatibility in Streptomyces. J. gen. Microbiol. 20, 442.

GarnJobst, L. \& Wilson, J. (1956). Heterocaryosis and protoplasmic incompatibility in Neurospora crassa. Proc. nat. Acad. Sci., Wash. 42, 613.

Hopwood, D. A. (1959). Linkage and the mechanism of recombination in Streptomyces coelicolor. Ann. N.Y. Acad. Sci. 81 (4), 887.

Kutzner, H. J. \& Waksman, S. A. (1959). Streptomyces coelicolor Muller and Streptomyces violaceoruber Waksman and Curtis, two distinctly different organisms. J. Bact. 78, 528.

Lederberg, J. \& Lederberg, E. M. (1952). Replica plating and indirect selection of bacterial mutants. J. Bact. 63, 399.

Lederberg, J., Cavalli, L. L. \& Lederberg, E. M. (1952). Sex compatibility in Escherichia coli. Genetics, 37, 720 .

SAITo, H. (1958). Heterocaryosis and genetic recombination in Streptomyces griseoflavus. Canad. J. Microbiol. 4, 571.

Sermonti, G. \& Spada-Sermonti, I. (1955). Genetic recombination in Streptomyces. Nature, Lond. 176, 121.

Sermonti, G. \& Spada-Sermonti, I. (1956). Gene recombination in Streptomyces coelicolor. J. gen. Microbiol. 15, 609.

Szybalski, W. \& Braendle, D. H. (1956). Genetic recombination in Streptomyces. Bact. Proc. 1956, 48. 